\title{
Assessment of Unmet Clinical Need in Type 2 Diabetic Patients on Conventional Therapy in the UK
}

\author{
Hayley Bennett · Phil McEwan · Klas Bergenheim • Jason Gordon
}

To view enhanced content go to www.diabetestherapy-open.com

Received: May 16, 2014 / Published online: September 4, 2014

(C) The Author(s) 2014. This article is published with open access at Springerlink.com

\section{ABSTRACT}

Introduction: Type 2 diabetes mellitus (T2DM) is an increasing problem worldwide and a leading risk factor for cardiovascular disease. As beta cell function declines, the management of T2DM typically comprises of escalations in treatment from diet and exercise to oral therapies and eventually insulin. Treatment algorithms based on the attainment of blood glucose targets may not account for changes in other cardiovascular risk factors. The objective

Electronic supplementary material The online version of this article (doi:10.1007/s13300-014-0079-6) contains supplementary material, which is available to authorized users.

H. Bennett $(\bowtie)$

Health Economics and Outcomes Research Ltd,

Singleton Court Business Park, Wonastow Rd,

Monmouth NP25 5JA, UK

e-mail: hayley.bennettwilton@heor.co.uk

P. McEwan · J. Gordon

Health Economics and Outcomes Research Ltd.,

Wales, UK

P. McEwan

Swansea Centre for Health Economics, Swansea

University, Wales, UK

K. Bergenheim

Global Health Economics and Outcomes Research,

AstraZeneca, Molndal, Sweden of this study is to describe unmet clinical need, defined as failure to reduce weight or meet targets for blood pressure, total cholesterol or glycated hemoglobin (HbA1c) levels.

Methods: Anonymized UK patient data for those (1) initiating oral antidiabetic drug (OAD) monotherapy, (2) escalating to dual therapy, (3) escalating to triple therapy, and (4) escalating to insulin therapy over the study period (01/01/2005-31/12/2009) were obtained from The Health Improvement Network (THIN). Changes in risk factors were evaluated before and after therapy escalation, and the attainment of targets, assessed at the last recorded measurement, as follows: HbA1c $<7.5 \%$, systolic blood pressure (SBP) $<140 \mathrm{mmHg}$, total cholesterol (TC) $<5 \mathrm{mmol} / \mathrm{L}$, and reduction in weight.

Results: Prior to therapy escalation, mean HbA1c in each subgroup exceeded $7.5 \%$ and was higher respective to the number of OADs being used (monotherapy: 8.03\%; double: 8.48\%; triple: $8.71 \%$ ). Insulin users displayed the highest HbA1c prior to treatment escalation (9.78\%). Following escalation, a decline in HbA1c was observed in all subgroups. By contrast, mean SBP and TC levels decreased 
prior to the addition of a second and third oral therapy. Consistent improvements following treatment escalation were not observed across the other risk factors following therapy escalation. Overall, the proportion of subjects that attained all four targets ranged from 3\% (monotherapy and insulin) to $6 \%$ (dual therapy).

Conclusion: The potential unmet clinical need among conventionally treated T2DM patients is significant, with respect to the control of blood glucose and other cardiovascular risk factors: SBP, TC, and weight. There clearly remains the need for new therapeutic approaches to alleviate the burden associated with T2DM.

Keywords: Routine clinical practice; Type 2 diabetes mellitus; Unmet need

\section{INTRODUCTION}

Diabetes is an increasing problem in both developed and developing countries, and is a leading risk factor for vascular disease [1]. Recent estimates indicate that the prevalence of type 2 diabetes mellitus (T2DM) in the UK is around $4.3 \%$ [2]. Rising obesity and the demographic shift to an older population suggest that this prevalence will increase.

The treatment of T2DM comprises a combination of lifestyle changes and drug therapy. Care pathways for people with T2DM aim to address a decline in beta cell function typically via escalation from diet and exercise regimens to oral therapies and eventually insulin [3]. Oral antidiabetic drugs (OADs) are the mainstay of therapy for many T2DM patients, but many do not achieve the optimal reductions in weight, blood pressure or glycated hemoglobin (HbA1c), and might benefit from additional therapy. Consensus algorithms advocate the addition of a second $\mathrm{OAD}$ or basal insulin to metformin monotherapy, based on their glucose-lowering properties $[4,5]$. While international organizations recommend a range of options for second-line therapy [5], the National Institute for Health and Care Excellence (NICE) recommends addition of a sulphonylurea (SU) as the preferred second-line option [4].

Recommended treatment algorithms that are based on the attainment of blood glucose targets may not account for changes in other cardiovascular risk factors in patients escalating from first-line monotherapy. This study aims to describe the unmet clinical need, defined as failure to reduce weight or meet targets for systolic blood pressure (SBP), total cholesterol (TC) or HbA1c levels in T2DM patients taking commonly prescribed OADs.

\section{METHODS}

A retrospective cohort study was conducted to summarize patient characteristics (numbers on treatment, age, sex, concomitant lipid or blood pressure-lowering therapy) to estimate changes in the following cardiovascular risk factors: HbA1c, weight, SBP, and TC; and to estimate proportions of patients achieving outcome targets (i.e., HbA1c <7.5\%) for each OAD therapy cohort: mono, dual, triple, and insulin therapy.

\section{Data Source}

This study used retrospective cohort data extracted from The Health Improvement Network (THIN) database [6]. THIN currently contains the records of 9.1 million patients (3.4 million active patients) collected from $\sim 500$ general practices across the UK. THIN is a 
suitable data source for this study, which focuses on an unselected group of diabetic patients from the general population, rather than a specialist study group. This study was granted ethical approval from the Scientific Review Committee (SRC) (Reference 11-023, July 04, 2011).

\section{Study Population}

The study cohort consists of men and women with a diagnosis of T2DM recorded during the study period: 01/01/2005-31/12/2009. The index date was defined as the date of the first diagnosis recorded within the study period. Patients aged $<25$ years at index date and/or with steroid-induced or gestational diabetes were excluded. Patients were required to have at least 365 days of follow-up pre- and post-first OAD prescription, at least one reading of SBP, weight and $\mathrm{HbA1c}$ in 365 days prior to the first prescription date and at least two readings in 365 days following the first prescription date. The study focused on conventional therapies of the time and as such patients taking novel agents (e.g., DPP-4 inhibitors) were excluded.

The population was divided into four OAD exposure subgroups: mono, dual, triple, and insulin. Mono included all patients with only one OAD prescribed during 365 days after first OAD prescription, with at least four scripts issued. Dual included patients from the point of addition of a second $\mathrm{OAD}$, with at least four scripts for both drugs during the 365 days after this date. Triple included patients from the point of addition of a third OAD, with at least four scripts for each of the three drugs during the 365 days after this date. Insulin users included patients from the point of addition of insulin therapy, with at least four scripts for insulin during the 365 days after this date. Patients could be included in more than one subgroup over the course of the study period, though not simultaneously, provided they met the membership criteria of each subgroup.

\section{Study Variables and Analysis}

Changes in the outcome (risk factor) variables (HbA1c, weight, SBP and TC) were analyzed over time in two ways. Firstly, the crude change in each outcome was calculated as the difference between the measurement immediately preceding initiation (monotherapy cohort) or prior to therapy addition (dual, triple, and insulin therapy cohorts), and the last recorded measurement in the period following OAD therapy initiation/ escalation. Secondly, the gradient of change was calculated for the pre- and post-periods as the ratio of the change in each outcome and time (days) between the two measurements. The gradient provides a sense of the trajectory of each outcome in the pre-and post-periods, and hence the significance of OAD therapy initiation or escalation in terms of the change in risk factor adjusted for variation in time between observations. Subjects were required to have both measurement points pre- and postchange to be included in either calculation.

Subjects in each therapy cohort were evaluated for attainment of outcome targets at last recorded measurement. For HbA1c, SBP, and $\mathrm{TC}$, values below 7.5\%, $140 \mathrm{mmHg}$, and $5 \mathrm{mmol} / \mathrm{L}$, respectively, indicated a favorable risk factor profile [7]. For weight, a negative coefficient for change indicated a favorable risk factor profile. Levels of target attainment (each variable alone and combination of all four) were evaluated for each therapy cohort.

All outcomes were analyzed descriptively using means, proportions, and standard deviations (SD). $P$ values were presented for all 
$t$ tests at a 5\% level of significance. All analyses were conducted in Stata version 13 [8].

This article does not contain any new studies with human or animal subjects performed by any of the authors.

\section{RESULTS}

\section{Results by Therapy Cohort}

A total of 36,942 T2DM patients treated with one or more pharmacological agent in UK general practice were identified in the THIN dataset, with an average age of $>60$. The majority used a single OAD (monotherapy; $n=23,626)$, with progressively fewer subjects using two (dual therapy; $n=7,230$ ) and three OADs (triple therapy; $n=1,612$ ). There were a further 4,474 insulin users. There was an apparent positive association between lipidlowering and blood pressure pharmacological therapies, and progression from a single to multiple OADs (Table 1).

All OAD cohorts were associated with an increase in mean $\mathrm{HbA1c}$ prior to OAD initiation (monotherapy) or escalation (dual and triple therapy), followed by an observed decline in mean HbA1c post-initiation/ escalation (Table 1; Fig. 1). Insulin users had the highest starting HbA1c levels (mean $\pm \mathrm{SD}$ : $9.78 \pm 1.94$ ). Among noninsulin users, HbA1c levels prior to OAD progression were highest in the triple therapy cohort $(8.71 \pm 1.19)$, followed by dual therapy $(8.48 \pm 1.28)$ and monotherapy $(8.03 \pm 1.24)$. Across all therapy cohorts, statistically significant $(P<0.05)$ changes in HbA1c between -0.93 and -1.47 were observed in the period following therapy progression.
Mean HbA1c levels observed in each OAD cohort before and after the initiation/escalation of therapy, plotted against the HbA1c profile predicted using results of the UK Prospective Diabetes Study (UKPDS) can be seen in Fig. 2 [9]; the position of reductions in HbA1c plotted for each OAD cohort reflects the mean duration of treatment observed in each cohort prior to therapy escalation. At initiation of monotherapy, the mean time since diagnosis of diabetes was $1.00 \pm 1.60$ years: patients received monotherapy for $2.64 \pm 2.06$ years prior to the addition of a second OAD; patients received dual therapy for $3.15 \pm 2.41$ years prior to the addition of a third OAD. The mean characteristics of the monotherapy cohort were used to initialize the HbA1c trajectory derived using the UKPDS 68 equation and therapy escalation thresholds implemented at 8.48 and $8.71 \%$, corresponding to the dual and triple therapy cohorts, respectively.

With regard to weight profile, the monotherapy cohort was associated with a decline in weight $(\mathrm{kg})$ of $-1.67 \pm 0.32$ $(P<0.001)$. Conversely, the addition of a second or third OAD or insulin was associated with weight gain at therapy escalation $(P<0.05)$.

With regard to the risk factors SBP and TC, the monotherapy cohort experienced an average reduction of $-1.96 \pm 0.10$ in SBP $(P<0.001)$ and $-0.52 \pm 0.02$ in $\mathrm{TC}$ $(P<0.001)$. Dual therapy subjects and insulin users were associated with a decrease in TC and increase in SBP, while the converse was observed in subjects adding a third OAD. Attainment of SBP and TC targets was low, despite the use of blood pressure and lipid-lowering medications in the majority of patients. 
Table 1 Summary of patient characteristics and risk factor analysis by OAD cohort

\begin{tabular}{|c|c|c|c|c|}
\hline & Monotherapy & Dual therapy & Triple therapy & Insulin \\
\hline \multicolumn{5}{|l|}{ Patient characteristics } \\
\hline Number of patients & 23,626 & 7,230 & 1,612 & 4,474 \\
\hline Age (years, SD) & $62.75(12.59)$ & $62.91(11.80)$ & $60.76(11.21)$ & $61.40(12.85)$ \\
\hline Sex (\% male) & 56.68 & 60.39 & 65.07 & 56.97 \\
\hline $\begin{array}{l}\text { Lipid-lowering therapy }{ }^{\mathrm{a}, \mathrm{b}} \text { (\%, prior to } \\
\text { initiation) }\end{array}$ & 54.38 & 80.07 & 83.62 & 79.21 \\
\hline $\begin{array}{l}\text { Blood pressure tablets }{ }^{\mathrm{a}, \mathrm{c}}(\% \text {, prior to } \\
\text { initiation) }\end{array}$ & 65.91 & 74.92 & 76.67 & 76.82 \\
\hline \multicolumn{5}{|l|}{ Risk factor analysis } \\
\hline \multicolumn{5}{|l|}{ HbAlc (\%) } \\
\hline Prior to initiation, mean (SD) & $8.03(1.24)$ & $8.48(1.28)$ & $8.71(1.19)$ & $9.78(1.94)$ \\
\hline After initiation, mean (SD) & $7.10(1.00)$ & $7.38(1.18)$ & $7.66(1.29)$ & $8.30(1.58)$ \\
\hline Difference, mean $(\mathrm{SD})$ ( $P$ value $)$ & $\begin{array}{l}-0.93(0.17) \\
(0.001)\end{array}$ & $\begin{array}{c}-1.1(0.02) \\
(<0.001)\end{array}$ & $\begin{array}{l}-1.05(0.05) \\
(<0.001)\end{array}$ & $\begin{array}{c}-1.47(0.04) \\
(<0.001)\end{array}$ \\
\hline \multicolumn{5}{|l|}{ Weight (kg) } \\
\hline Prior to initiation, mean (SD) & $91.32(89.65)$ & $90.05(19.17)$ & $90.75(19.67)$ & $87.12(19.21)$ \\
\hline After initiation, mean (SD) & $89.65(20.02)$ & $91.05(20.06)$ & $93.28(20.84)$ & $89.24(19.39)$ \\
\hline Difference, mean $(\mathrm{SD})$ ( $P$ value $)$ & $\begin{array}{c}-1.67(0.32) \\
(<0.001)\end{array}$ & $\begin{array}{l}1.00(0.46) \\
(0.032)\end{array}$ & $\begin{array}{l}2.53(0.83) \\
(0.002)\end{array}$ & $\begin{array}{l}2.12(0.48) \\
\quad(<0.001)\end{array}$ \\
\hline \multicolumn{5}{|l|}{ Blood pressure (SBP) } \\
\hline Prior to initiation, mean (SD) & $139.47(16.95)$ & $136.37(15.67)$ & $136.89(15.28)$ & $135.32(17.39)$ \\
\hline After initiation, mean (SD) & $137.51(16.87)$ & $136.79(16.28)$ & $135.83(16.06)$ & $136.39(17.88)$ \\
\hline Difference, mean (SD) ( $P$ value $)$ & $\begin{array}{c}-1.96(0.19) \\
(<0.001)\end{array}$ & $\begin{array}{c}0.43(0.32) \\
(0.177)\end{array}$ & $\begin{array}{c}-1.06(0.58) \\
(0.069)\end{array}$ & $\begin{array}{c}1.07(0.39) \\
(0.006)\end{array}$ \\
\hline \multicolumn{5}{|l|}{ Total cholesterol (TC) } \\
\hline Prior to initiation, mean (SD) & $4.79(1.16)$ & $4.23(0.93)$ & $4.14(0.87)$ & $4.41(1.18)$ \\
\hline After initiation, mean (SD) & $4.27(0.96)$ & $4.06(0.91)$ & $4.16(0.91)$ & $4.13(1.05)$ \\
\hline Difference, mean (SD) ( $P$ value $)$ & $\begin{array}{c}-0.52(0.02) \\
(<0.001)\end{array}$ & $\begin{array}{c}-0.17(0.02) \\
(<0.001)\end{array}$ & $\begin{array}{c}0.02(0.04) \\
(0.484)\end{array}$ & $\begin{array}{c}-0.28(0.03) \\
(<0.001)\end{array}$ \\
\hline \multicolumn{5}{|c|}{ Proportion of subjects achieving target (mean) (\%): } \\
\hline HbAlc $<7.5 \%$ & 27 & 56 & 46 & 26 \\
\hline Weight decline at last measurement & 17 & 33 & 26 & 24 \\
\hline SBP $<140$ & 37 & 53 & 51 & 52 \\
\hline $\mathrm{TC}<5.0$ & 30 & 41 & 60 & 57 \\
\hline
\end{tabular}


Table 1 continued

\begin{tabular}{lllll}
\hline & Monotherapy & Dual therapy & Triple therapy & Insulin \\
\hline All targets reached & 3 & 6 & 5 & 3 \\
\hline
\end{tabular}

HbAlc glycated hemoglobin, $O A D$ oral antidiabetic drug, $S B P$ systolic blood pressure, $S D$ standard deviation, $T C$ total cholesterol

a Prior to initiation

b Lipid-lowering therapies used by $>5 \%$ patients receiving therapy: nicotinic acid, simvastatin, fenofibrate, cerivastatin, atorvastatin, pravastatin, and rosuvastatin

c Blood pressure medications used by $>5 \%$ patients receiving therapy: diltiazem, nifedipine, chlorothiazide + spironolactone, verapamil, captopril, furosemide, Ramipril, chlorothiazide, enalapril, chlorothiazide + spironolactone + lactose, co-amilofruse
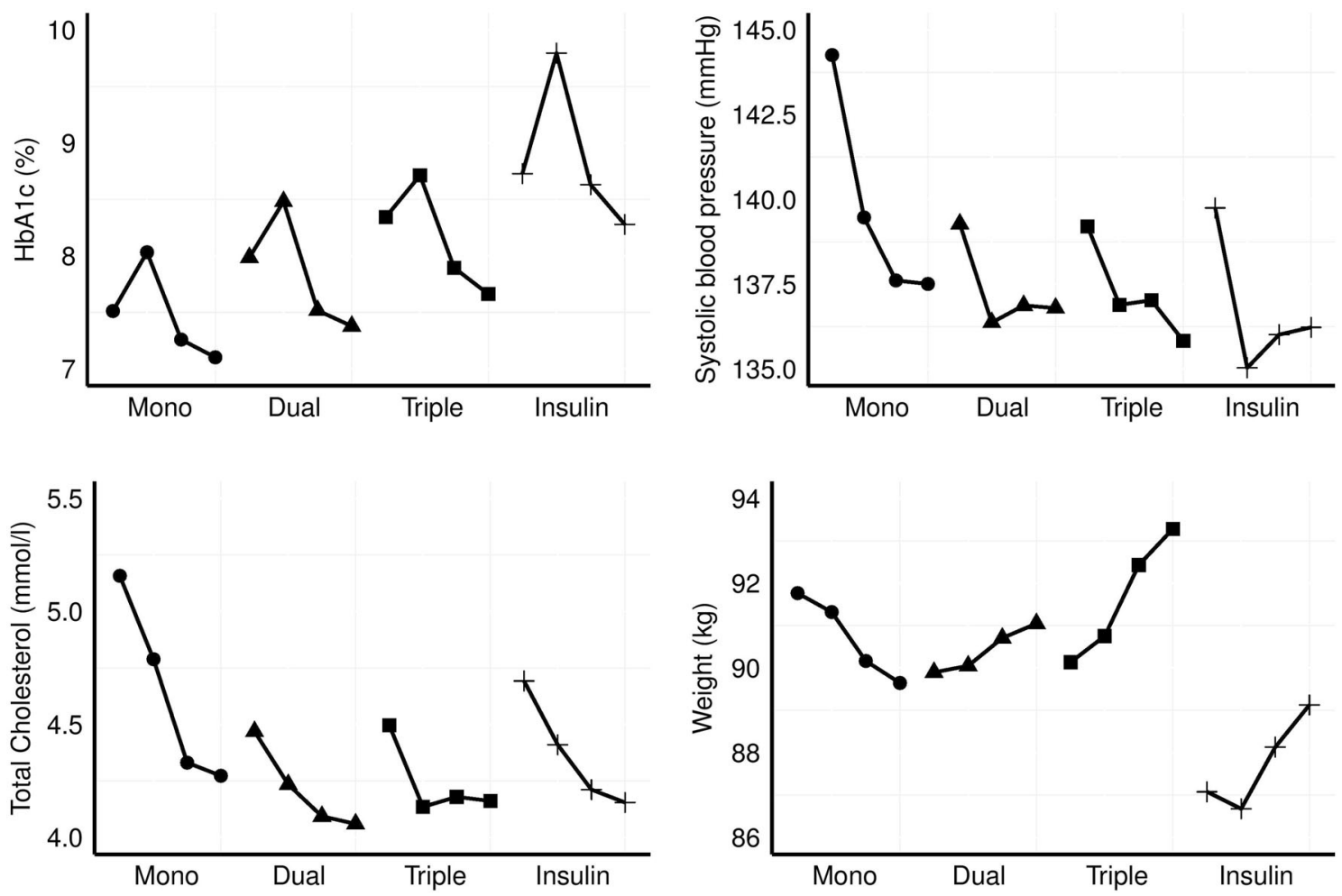

Fig. 1 Risk factor profiles before (1st and 2nd points) and after (3rd and 4th points) therapy escalation for each patient cohort

Overall, the proportion of subjects that attained targets across all four risk factors (HbA1c, weight, SBP, TC) was highest in the dual therapy cohort $(6 \%)$, followed by triple therapy (5\%) and monotherapy (3\%). Target attainment among insulin users was 3\% on average, similar to the monotherapy group (Table 1).

\section{Results by OAD Combination Within Therapy Cohort}

The majority of monotherapy subjects used metformin (89.59\%) followed by an SU (glibenclamide, gliclazide, glimepiride, glipizide or tolbutamide; 9.77\%), a thiazolidinedione (TZD; 0.58\%) and other 


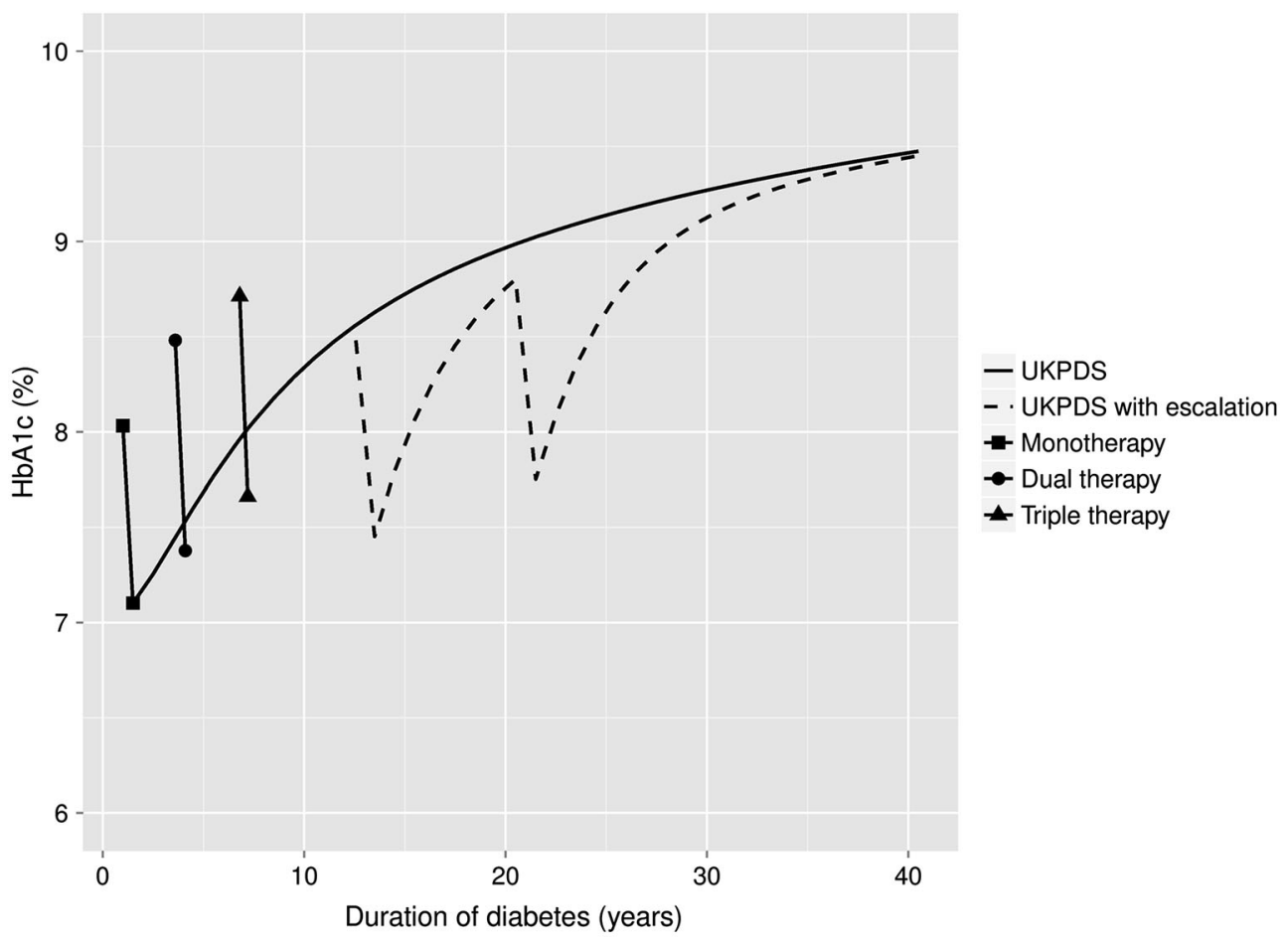

Fig. 2 Mean HbAlc levels observed in THIN before and after OAD therapy initiation/escalation versus HbA1c progression predicted by the UKPDS 68 equation with and without therapy escalation at corresponding $\mathrm{HbAlc}$

OADs (acarbose or meglitinide [repaglinide or nateglinide]; $0.06 \%)$. SU users were the oldest group (69.82 \pm 13.19$)$, associated with the greatest improvement in mean HbA1c $(-1.18 \pm 0.06, P<0.001)$; all OAD groups were associated with a reduction in HbA1c. Though other monotherapy users gained weight, the initiation of metformin was associated with a reduction in weight of $-1.98 \pm 0.33, P<0.001$ (SDC Table 1). SBP generally decreased for each of the OADs, except for SU which was associated with an increase of $0.41 \pm 0.68, P=0.541$. A reduction in TC was observed for each of the main OAD types $(P<0.001$, except for TZD users). No more than $3 \%$ of patients attained all four targets in each OAD group. Rates of achievement were consistently lowest for the weight target (7-18\%). The TZD group had the highest achievement of individual outcomes, thresholds; 8.48 and $8.71 \%$. HbAlc, glycated hemoglobin; $O A D$, oral antidiabetic drugs; THIN, The Health Improvement Network; UKPDS, UK Prospective Diabetes Study

with the exception of TC: $39 \%$ for HbA1c, $18 \%$ for weight, $54 \%$ for SBP, and $26 \%$ for TC.

Following monotherapy, the addition of SU to metformin accounted for $55.34 \%$ of all dual therapy subjects (SDC Table 2). SU added to metformin was associated with a reduction in $\mathrm{HbA1c}$ of $-1.13 \pm 0.04 \quad(P<0.001)$ and weight gain of $1.28 \pm 0.60 \quad(P=0.033) ; \mathrm{SBP}$ increased $(0.76 \pm 0.42, \quad P=0.066)$, and TC was reduced by $-0.21 \pm 0.03 \quad(P<0.001)$. Following the addition of SU, $6 \%$ of metformin subjects achieved all four outcomes targets, compared to $7 \%$ in $\mathrm{SU}$ users who added metformin, and $7 \%$ in metformin users who added TZD.

In subjects progressing to a third OAD, results were consistent with respect to the direction of change for each outcome for each of the OAD types analyzed (SDC Table 3). 
Reductions in HbA1c ranged from -0.90 to $-1.33(P<0.001$ in each group), increases in weight from 2.12 to $3.63 \quad(P<0.05$ in metformin + SU + TZD group only), decreases in SBP from -0.48 to $-2.35 \quad(P<0.05$ in $\mathrm{SU}+$ metformin $+\mathrm{TZD}$ group only), and increases in TC from 0.11 to $0.25(P<0.05$ in each group, with the exception of metformin $+S U+$ TZD). Proportions of patients attaining all four targets ranged from $4 \%$ to $8 \%$ in the metformin + SU + TZD and metformin + TZD + SU groups, respectively.

Among the 4,474 insulin users, 4,464 (99.8\%) used at least one OAD, 3,730 (83.4\%) used two OADs and 1,358 (30.4\%) used three OADs. Age, sex, and concomitant lipid-lowering and blood pressure therapy use were similar between the groups at baseline (SDC Table 4). HbA1c and TC levels consistently fell among insulin users $(P<0.001)$, while weight $(P<0.05)$ and SBP $(P<0.01)$ increased in all the three OAD groups. Similar proportions of patients achieved target attainment in each OAD/ insulin group (1-3\%).

\section{Results by Age}

Subgroup analyses of patients above and below 70 years of age did not suggest that levels of target attainment were lower in older patients (SDC Table 5). With the exception of SBP in patients receiving more than one $\mathrm{OAD}$, observed attainment rates of individual and combined targets were not lower in the higher age group.

\section{DISCUSSION}

Unmet clinical need may be broadly defined as a "medical need that is not addressed adequately by an existing therapy" [10]. In the context of pharmacological management of T2DM, this definition relates to treatments that do not improve or adequately control risk factors. Previous studies have demonstrated delays in treatment intensification in UK clinical practice despite poor glycemic control [11]. In this study, the cardiovascular risk factors, weight, SBP, and TC, were considered in addition to glycemic control, the predominant measure of success of pharmacotherapy in this setting [10].

Using routinely collected patient-level data from UK general practice, a potential unmet clinical need was identified, in the form of patients requiring progression to multiple pharmacotherapies and a significant proportion of patients failing to achieve reductions in the risk factors considered to be the goals of therapy across all pathways examined.

People with T2DM treated with a single OAD were associated with improvements in each of the four risk factors analyzed (HbA1c, weight, SBP and TC), but relative to dual and triple therapy users, fewer monotherapy subjects attained target levels for each of these risk factors; consequently, single $\mathrm{OAD}$ use was associated with the lowest proportion of subjects achieving a reduction in all four risk factors. Insulin users demonstrated reductions in HbA1c and TC, but an increase in weight and SBP.

HbA1c levels prior to therapy initiation/ escalation were higher with the addition of each $\mathrm{OAD}$, and were higher again in insulin users. This might reflect a decline in beta cell function that is associated with the natural progression of T2DM, and hence the need for more intensive pharmacological management [3]. A pattern of increased or decreased starting levels from single to multiple OAD therapies was not observed for weight. However, for both 
SBP and TC, levels prior to initiation of monotherapy were higher than levels prior to escalation to a second and third OAD.

Together, these findings indicate that in UK general practice, therapy escalation in the management of T2DM is triggered by the need for better glycemic control (as evidenced by higher starting levels prior to change). It may be inferred that SBP and TC, conversely, do not drive the timing of therapy escalation, as evidenced by the lower starting levels for these outcomes in the multiple OAD cohorts, though they may influence the choice of additional OAD at therapy escalation.

The 2012 quality outcomes framework (QoF) results suggested that attainment levels of HbA1c targets $(\leq 7 \%)$ in England and Wales were approaching 70\%. Attainment of blood pressure and cholesterol targets were $\sim 71 \%$ and $82 \%$, respectively $[12,13]$. Measures such as QoF paint a picture of successful T2DM management with respect to the control of cardiovascular risk factors and the satisfaction of related targets; however, results presented herein suggest that this is not the case for the many patients requiring therapy escalation in clinical practice.

Of the four targets evaluated, lowest attainment levels were observed for weight. Some glucose-lowering therapies are associated with weight gain, which may contribute to these observations. Weight change was included in this study despite this link due to its importance as a recognized cardiovascular risk factor, associated with excess mortality risk [14] and negative impact on quality of life [15].

To support economic evaluation, many T2DM disease models utilize results from UKPDS, including predicting the expected trajectory of HbA1c levels [9]. However, realworld observations suggest that the HbA1c profiles of patients undergoing treatment escalation do not conform to this average predicted trajectory, and additional OADs may be added sooner than is predicted. A possible explanation for this may be that there exists a group of slow progressing patients, whose HbA1c is well controlled, bringing the average trajectory of HbA1c down and distorting our understanding of reality. The observed numbers of patients in each cohort would support this idea; with the majority of patients captured in THIN receiving monotherapy and smaller patient groups progressing to double, triple or insulin therapy.

From the patients' perspectives, suboptimal clinical response is known to increase morbidity and mortality, and reduce quality of life, from a range of health-related complications. In turn, sufferers and their families are financially burdened, most commonly with out-of-pocket expenses associated with disease management and losses in earning potential [16]. The economic implications from such unmet need are considerable; recent research has estimated that the true cost of managing T2DM in the UK is in the region of $£ 3.5$ billion [17, 18]. Hence, newer treatments for T2DM and combinations thereof that have been shown to be clinically effective (in absolute and comparative terms) are required to further improve patient health outcomes and reduce the economic burden of T2DM.

The findings of this research are set against a number of inherent limitations in the use of routinely collected observational data in characterizing patient outcomes following treatment. These include treatment selection bias where factors that have determined what type(s) of pharmacotherapy an individual receives are not accounted for in the analysis. On this limitation, the aim of this study was to statistically describe the observed data; as such, statistical techniques to (potentially) address 
such bias were not employed at the data extraction or analysis stage, though such techniques cannot fully account for the inherent limitations of datasets such as THIN. It is considered, however, that the value of these analyses outweighs this limitation [19]. In line with the audit-style approach of the study, the significance of missing records among routinely collected data was not investigated.

This study was a cross-sectional analysis of patient cohorts defined by the number of and timing of pharmacotherapies used in the management of T2DM. It was not possible to map the trajectory of individual patients as they moved from single to multiple OADs and to insulin therapy.

Treatment goals are highly individualized in practice. Though clinicians may take a more cautious approach to the management of elderly patients, results did not suggest that target attainment was lower in patients aged $>70$ years. While lower HbA1c targets of $6.5 \%$ or $7 \%$ may be appropriate for some patients, $7.5 \%$ was evaluated as a less stringent target relevant to the majority of patients across all lines of therapy. Consequently, reported attainment of $\mathrm{HbA1c}$ targets may be overestimated among patients at low risk of hypoglycemia, with short duration of diabetes and little comorbidity. Though it was outside the scope of this study, the incidence of hypoglycemia and hospitalization are important patient outcomes for consideration, particularly following therapy initiation and intensification.

This study focused on unmet need among patients receiving conventional therapies. A future evaluation could assess whether similar levels of unmet need exist, and whether the observed therapy escalation thresholds remain, among patients prescribed novel therapies such as DPP-4 inhibitors.
The explanations underlying instances of suboptimal clinical outcomes and poor target attainment characterizing unmet clinical need in this population cannot be inferred with certainty.

\section{CONCLUSION}

Patient outcomes among people with T2DM treated in UK general practice were often below what are considered to be milestones of optimal clinical response, even with a combination of pharmacotherapies. The potential unmet clinical need among people with T2DM is significant with respect to the control of blood glucose and other cardiovascular risk factors, including TC, SBP, and weight. Conventional treatment strategies are variably effective in managing the condition, and thus there is a need for new therapeutic agents, or approaches to treatment, to alleviate the health and economic burden associated with the T2DM.

\section{ACKNOWLEDGMENTS}

Sponsorship and article processing charges for this study were funded by AstraZeneca plc, Molndal, Sweden. The funding agreement ensured the authors' independence in designing the study, interpreting the data, writing, and publishing the report. Editorial assistance for this study was provided by Samantha Webster of Health Economics and Outcomes Research Ltd and funded by AstraZeneca plc, Molndal, Sweden. All named authors meet the ICMJE criteria for authorship for this manuscript, take responsibility for the integrity of the work as a whole, and have given final approval to the version to be published. 
Conflict of interest. Klas Bergenheim is an employee of AstraZeneca plc.

Compliance with ethics. This article does not contain any new studies with human or animal subjects performed by any of the authors.

Open Access. This article is distributed under the terms of the Creative Commons Attribution Noncommercial License which permits any noncommercial use, distribution, and reproduction in any medium, provided the original author(s) and the source are credited.

\section{REFERENCES}

1. American Diabetes Association, Lung National Heart, and Blood Institute, Juvenile Diabetes Foundation International, National Institute of Diabetes and Digestive and Kidney Diseases, American Heart Association. Diabetes mellitus: a major risk factor for cardiovascular disease. Circulation. 1999;100:1132-3.

2. Massó González EL, Johansson S, Wallander M-A, García Rodríguez LA. Trends in the prevalence and incidence of diabetes in the UK: 1996-2005. J Epidemiol Commun Health. 2009;63(4):332-6.

3. Festa A, Williams K, D'Agostino R Jr, Wagenknecht LE, Haffner SM. The natural course of beta-cell function in nondiabetic and diabetic individuals: the Insulin Resistance Atherosclerosis Study. Diabetes. 2006;55(4):1114-20.

4. National Institute for Health and Care Excellence. CG87. Type 2 diabetes-newer agents. 2009.

5. Inzucchi SE, Bergenstal RM, Buse JB, Diamant M, Ferrannini E, Nauck $M$, et al. Management of hyperglycemia in type 2 diabetes: a patientcentered approach: position statement of the American Diabetes Association (ADA) and the European Association for the Study of Diabetes (EASD). Diabetes Care. 2012;35(6):1364-79.

6. Cegedim strategic data. The Health Improvement Network (THIN) database. 2014 Accessed.

7. National Institute for Health and Care Excellence. CG66. Type 2 diabetes-the management of type 2 diabetes. 2008.
8. StataCorp. Stata Statistical Software: release 13. 2013.

9. Clarke PM, Gray AM, Briggs A, Farmer AJ, Fenn P, Stevens RJ, et al. A model to estimate the lifetime health outcomes of patients with type 2 diabetes: the United Kingdom Prospective Diabetes Study (UKPDS) Outcomes Model (UKPDS no. 68). Diabetologia. 2004;47(10):1747-59.

10. Food and drug administration. SOPP 8414: fast track drug development programs: designation and review programs. 2001. Available at http://www. fda.gov/biologicsbloodvaccines/guidancecomplianceregulatoryinformation/proceduressopps/ucm073515.htm. Accessed August 2014.

11. Khunti K, Wolden ML, Thorsted BL, Andersen M, Davies MJ. Clinical inertia in people with type 2 diabetes: a retrospective cohort study of more than 80,000 people. Diabetes Care. 2013;36(11):3411-7.

12. Health and Social Care Information Centre. Quality and outcomes framework-2011-12, England level. 2012. Available at http://www.hscic.gov.uk/ catalogue/PUB08661. Accessed August 2014.

13. StatsWales. Quality and outcomes framework. 2013. Available at https://statswales.wales.gov.uk/ Catalogue/Health-and-Social-Care/NHS-Primaryand-Community-Activity/GMS-Contract. Accessed August 2014.

14. Bodegard J, Sundstrom J, Svennblad B, Ostgren CJ, Nilsson PM, Johansson G. Changes in body mass index following newly diagnosed type 2 diabetes and risk of cardiovascular mortality: a cohort study of 8486 primary-care patients. Diabetes Metab. 2013;39(4):306-13.

15. Lane S, Levy AR, Mukherjee J, Sambrook J, Tildesley $H$. The impact on utilities of differences in body weight among Canadian patients with type 2 diabetes. Curr Med Res Opin. 2014;30(7):1267-73.

16. Kankeu HT, Saksena $\mathrm{P}, \mathrm{Xu} \mathrm{K}$, Evans DB. The financial burden from non-communicable diseases in low- and middle-income countries: a literature review. Health Res Policy Syst/BioMed Central. 2013;11:31.

17. NHS Diabetes. Report number 46357 Diabetes in the NHS, 2007. Published by the National Diabetes Support Team on behalf a group of diabetes specialists in England. 2007. Available at www. diabetes.nhs.uk/document.php?o=318. Accessed October 2011.

18. The NHS Information Centre. Prescribing for diabetes in England: 2005/6-2010/11. 2011. Available at http://www.ic.nhs.uk/webfiles/ publications/prescribing\%20diabetes\%20200506 
\%20to\%20201011/Prescribing_for_Diabetes_in England_20056_to_201011.pdf. Accessed October 2011.

19. Gordon J, Pockett RD, Tetlow AP, McEwan P, Home PD. A comparison of intermediate and long-acting insulins in people with type 2 diabetes starting insulin: an observational database study. Int J Clin Pract. 2010;64(12):1609-18. 